\title{
Higher order cohomological restrictions on anomalies and counterterms
}

\author{
G. Barnich* \\ Freie Universität Berlin, Fachbereich Physik, Institut für Theoretische \\ Physik, Arnimallee 14, D-14195 Berlin.
}

\begin{abstract}
Using a regularization with the properties of dimensional regularization, higher order local consistency conditions on one loop anomalies and divergent counterterms are given. They are derived without any a priori assumption on the form of the BRST cohomology and can be summarized by the statements that (i) the antibracket involving the first order divergent counterterms, respectively the first order anomaly, with any BRST cocycle is BRST exact, (ii) the first order divergent counterterms can be completed into a local deformation of the solution of the master equation and (iii) the first order anomaly can be deformed into a local cocycle of the deformed solution.
\end{abstract}

$\left.{ }^{*}\right)$ Alexander von Humboldt fellow. Starting November 1st 1997: Chargé de Recherches du Fonds National Belge de la Recherche Scientifique at Université Libre de Bruxelles, Faculté des Sciences, Campus Plaine C.P. 231, B-1050 Bruxelles, Belgium. 
Cohomological techniques in renormalization theory have attracted a lot of interest since their introduction in the pioneering work by Becchi, Rouet and Stora [1] in the context of Yang-Mills theories, because they allow to address general problems of perturbative quantum field theories, like the form of anomalies or of divergent counterterms by purely algebraic means (see [2] for a recent review). The BRST construction and the formulation of Zinn-Justin thereof in terms of the generating functional for vertex functions [3] has then been generalized to theories with general local symmetries by Batalin and Vilkovisky [4].

In this more general setting, one loop anomalies are constrained by the cohomology groups in ghost number 1 of the BRST differential generated by a solution to the master equation [5, 6]. This represents the generalization of the Wess-Zumino consistency condition [7] for the case of the gauge anomaly. The cohomological restrictions on the one loop divergent counterterms, involving the ghost number 0 group, have been discussed in [8, 9], where it is stressed that these techniques are valid in the power counting non renormalizable case or for the case of higher dimensional operators (see also [10]) and hence apply to effective field theories [11].

These works only needed the first order consistency conditions following from the quantum action principle mainly for the following reason. One can show [12] that for semi-simple Yang-Mills theory or gravity, the cohomology groups in ghost number 0 and 1 can be described independently of the antifields, so that all higher order constraints to be found below turn out to be trivial. This is however not the case in general, where the form of the BRST cohomology groups in ghost number 0 and 1 can be more involved. In this case, higher order considerations will be relevant.

The purpose of this letter is to give a purely cohomological description in the space of local functionals of the higher order restrictions on the one loop anomalies and counterterms in terms of deformation theory. To derive these conditions, we assume that there is a regularization scheme with the properties of dimensional regularization as used in [13], although we expect the cohomological restrictions to be independent of the regularization method. Notational conventions for the Batalin-Vilkovisky formalism will be those of the reviews [14, 15]. The analysis applies to local and rigid symmetries if we understand the master equation to be the generalized master equation discussed in [16]. In this letter, only the cocyle condition is considered. More details including a discussion on the coboundary conditions will appear 
elsewhere [17].

A different, but related issue is the problem of cohomological restrictions on anomalies and counterterms at higher loops. Such considerations have appeared in the recent literature [18, 13, 19] from various points of view, and in particular, the form of the consistency conditions at higher loops has been discussed in 20] in the context of non local regularization. These issues will be adressed in the set-up of this letter in [17].

\section{Regularization}

We will assume that there is a regularization with the properties of dimensional regularization as explained in reference [13], i.e.,

- the regularized action $S_{\tau}=\Sigma_{n=0} \tau^{n} S_{n}$ is a polynomial or a power series in $\tau$, the $\tau$ independent part corresponding to the starting point action $S_{0}=S$,

- if the renormalization has been carried out up to $n-1$ loops, the divergences of the effective action at $n$ loops are poles in $\tau$ up to the order $n$ with residues that are local functionals, and

- the regularized quantum action principle holds [21].

Let $\tilde{S}=S_{\tau}+\rho^{*} \theta_{\tau}$, with $\theta_{\tau}=\frac{1}{2 \tau}\left(S_{\tau}, S_{\tau}\right)$, so that $\theta_{0}=\left(S, S_{1}\right)$, and $\rho^{*}$ a global source in ghost number -1 . On the classical level, we have, using $\left(\rho^{*}\right)^{2}=0$,

$$
\begin{array}{r}
\frac{1}{2}(\tilde{S}, \tilde{S})=\tau \frac{\partial \tilde{S}}{\partial \rho^{*}} \\
\left(\tilde{S}, \frac{\partial \tilde{S}}{\partial \rho^{*}}\right)=0
\end{array}
$$

which translates, according to the quantum action principle, into the corresponding equations for the regularized generating functional for 1PI vertex functions:

$$
\begin{array}{r}
\frac{1}{2}(\tilde{\Gamma}, \tilde{\Gamma})=\tau \frac{\partial \tilde{\Gamma}}{\partial \rho^{*}} \\
\left(\tilde{\Gamma}, \frac{\partial \tilde{\Gamma}}{\partial \rho^{*}}\right)=0
\end{array}
$$


Using $\left(\rho^{*}\right)^{2}=0$, these equations reduce to

$$
\begin{array}{r}
\frac{1}{2}(\Gamma, \Gamma)=\tau \frac{\partial \tilde{\Gamma}}{\partial \rho^{*}} \\
\left(\Gamma, \frac{\partial \tilde{\Gamma}}{\partial \rho^{*}}\right)=0 .
\end{array}
$$

At one loop, we get

$$
\begin{array}{r}
\left(S_{\tau}, \Gamma^{(1)}\right)=\tau \theta^{(1)}, \\
\left(S_{\tau}, \theta^{(1)}\right)+\left(\Gamma^{(1)}, \theta_{\tau}\right)=0,
\end{array}
$$

where $\Gamma^{(1)}$ and $\theta^{(1)}$ are respectively the one loop contributions of $\Gamma$ and $\partial \tilde{\Gamma} / \partial \rho^{*}$. By assumption, we have

$$
\begin{gathered}
\Gamma^{(1)}=\sum_{n=-1} \tau^{n} \Gamma^{(1) n}, \\
\theta^{(1)}=\sum_{n=-1} \tau^{n} \theta^{(1) n},
\end{gathered}
$$

where $\Gamma^{(1)-1}$ and $\theta^{(1)-1}$ are local functionals.

\section{Lowest order cohomological restrictions}

At $1 / \tau$, equations (1.7) and (1.8) give

$$
\begin{aligned}
\left(S, \Gamma^{(1)-1}\right) & =0, \\
\left(S, \theta^{(1)-1}\right)+\left(\Gamma^{(1)-1}, \theta_{0}\right) & =0 .
\end{aligned}
$$

Using $\theta_{0}=\left(S, S_{1}\right)$ and equation (2.1), equation (2.2) reduces to

$$
\left(S, \theta^{(1)-1}-\left(S_{1}, \Gamma^{(1)-1}\right)\right)=0 .
$$

In addition, we get, from the term independent of $\tau$ in equation (1.7),

$$
\left(S, \Gamma^{(1) 0}\right)=\theta^{(1)-1}-\left(S_{1}, \Gamma^{(1)-1}\right) .
$$

The term linear in $\tau$ gives

$$
\left(S, \Gamma^{(1) 1}\right)=\theta^{(1) 0}-\left(S_{1}, \Gamma^{(1) 0}\right)-\left(S_{2}, \Gamma^{(1)-1}\right) .
$$


The one loop renormalized effective action is $\Gamma_{R}^{1}=S+\hbar \Gamma^{(1) 0}+O\left(\hbar^{2}, \tau\right)$, where the notation $O\left(\hbar^{2}, \tau\right)$ means that those terms which are not of order at least two in $\hbar$ are of order at least one in $\tau$ and vanish when the regularization is removed $(\tau \longrightarrow 0)$, so that

$$
\frac{1}{2}\left(\Gamma_{R}^{1}, \Gamma_{R}^{1}\right)=\hbar A_{1}+O\left(\hbar^{2}, \tau\right),
$$

with, using equation (2.4),

$$
A_{1}=\theta^{(1)-1}-\left(S_{1}, \Gamma^{(1)-1}\right),
$$

and the consistency conditions for local functionals

$$
\begin{array}{r}
\left(S, \Gamma^{(1)-1}\right)=0 \Longrightarrow \Gamma^{(1)-1}=c_{1}^{i} C_{i}+\left(S, \Xi_{1}\right), \\
\left(S, A_{1}\right)=0 \Longrightarrow A_{1}=a_{1}^{i} A_{i}+\left(S, \Sigma_{1}\right),
\end{array}
$$

where $C_{i}$ and $A_{i}$ are respectively a basis of representatives for $H^{0}(s)$ and $H^{1}(s)$.

\section{First order cohomological restrictions}

Let $D$ be a BRST cocycle in any ghost number $g$ and consider $S^{j}=S+j D$, where the source $j$ is of ghost number $-g$. The regularized action is $S_{\tau}^{j}=$ $S_{\tau}+j D_{\tau}$, with $D$ a polynomial in $\tau$ starting with $D$. If $\tilde{S}^{j}=S_{\tau}^{j}+\rho^{*} \theta_{\tau}^{j}$, where $\theta_{\tau}^{j}=\frac{1}{2 \tau}\left(S_{\tau}, S_{\tau}\right)+\frac{1}{\tau}\left(j D_{\tau}, S_{\tau}\right)$, we have

$$
\frac{1}{2}\left(\tilde{S}^{j}, \tilde{S}^{j}\right)=\tau \frac{\partial \tilde{S}^{j}}{\partial \rho^{*}}+O\left(j^{2}\right),
$$

and the corresponding equation for the regularized generating functional $\tilde{\Gamma}_{j}$

$$
\frac{1}{2}\left(\tilde{\Gamma}_{j}, \tilde{\Gamma}_{j}\right)=\tau \frac{\partial \tilde{\Gamma}_{j}}{\partial \rho^{*}}+O\left(j^{2}\right) .
$$

At one loop, we get for the term independent of $\rho^{*}$,

$$
\left(\Gamma_{j}^{(1)}, S_{\tau}^{j}\right)=\tau \theta_{j}^{(1)}+O\left(j^{2}\right) .
$$

The term linear in $j$ of order $\frac{1}{\tau}$ gives

$$
\left(D^{(1)-1}, S\right)+\left(D, \Gamma^{(1)-1}\right)=0,
$$

with $D^{(1)-1}=\left.\left(\partial \Gamma_{j}^{(1)-1} / \partial j\right)\right|_{j=0}$. This gives our first theorem. 
Theorem 3.1 The antibracket of the divergent one loop part $\Gamma^{(1)-1}$, which is BRST closed and local, with any local BRST cocycle is BRST exact in the space of local functionals.

The theorem can be reformulated by saying that the antibracket map (induced in the local BRST cohomology groups by the antibracket, see ref. [22])

$$
\left(\left[\Gamma^{(1)-1}\right],[D]\right)=[0]
$$

for all $[D] \in H^{g}(s)$. This equation represents a cohomological restriction on the coefficients $c_{1}^{i}$ that can appear ; it can be calculated classically from the knowledge of $H^{0}(s)$ and the antibracket map from $H^{0}(s) \times H^{g}(s)$ to $H^{g+1}(s)$. According to the previous section, the theorem holds in particular when $D=\Gamma^{(1)-1}$ or $D=A_{1}$.

In the same way, the consistency condition is

$$
\left(\Gamma_{j}, \frac{\partial \tilde{\Gamma}_{j}}{\partial \rho^{*}}\right)+O\left(j^{2}\right)=0
$$

and gives at one loop,

$$
\left(\Gamma_{j}^{(1)}, \theta_{\tau}^{j}\right)+\left(S_{\tau}^{j}, \theta_{j}^{(1)}\right)+O\left(j^{2}\right)=0 .
$$

The term linear in $j$ of order $\frac{1}{\tau}$ gives

$$
\begin{array}{r}
\left(D^{(1)-1}, \theta_{0}\right)-\left(\left.\frac{\partial \theta_{0}^{j}}{\partial j}\right|_{j=0}, \Gamma^{(1)-1}\right) \\
+\left(D, \theta^{(1)-1}\right)-\left(\left.\frac{\partial \theta_{j}^{(1)-1}}{\partial j}\right|_{j=0}, S\right)=0 .
\end{array}
$$

Using $\theta_{0}=\left(S, S_{1}\right), \partial \theta_{0}^{j} /\left.\partial j\right|_{j=0}=\left(D_{1}, S\right)+\left(D, S_{1}\right)$, equations (2.1), (2.7) and (3.4), we get

$$
\left(D, A_{1}\right)-\left(\left.\frac{\partial \theta_{j}^{(1)-1}}{\partial j}\right|_{j=0}-\left(D_{1}, \Gamma^{(1)-1}\right)-\left(D^{(1)-1}, S_{1}\right), S\right)=0 .
$$

This gives our second result. 
Theorem 3.2 The antibracket of the BRST closed first order anomaly $A_{1}$ with any local BRST cocycle is BRST exact in the space of local functionals.

The theorem can again be reformulated by saying that the antibracket map

$$
\left(\left[A_{1}\right],[D]\right)=[0]
$$

for all $[D] \in H^{g}(s)$; it represents a classical cohomological restriction on the coefficients $a_{1}^{i}$ that can appear.

\section{Higher orders}

Let $B^{0}=S$ and $B^{1}=\Gamma^{(1)-1}$. We have the following theorem.

Theorem 4.3 The first order counterterms can be completed into a local deformation of $S$, i.e., there exist local functionals $B^{n}$ such that

$$
\begin{array}{r}
\frac{1}{2}\left(S^{j^{\infty}}, S^{j^{\infty}}\right)=0, \\
S^{j^{\infty}}=S+\sum_{n=1} j^{n} B^{n} .
\end{array}
$$

The higher order cohomological restrictions of such an equation in terms of Lie-Massey brackets is briefly discussed in [22. More details will be given in [17].

Proof. The theorem is true for $j^{0}, j^{1}$ and $j^{2}$, if we take $D=\Gamma^{(1)-1}=B^{1}$ in (3.4) and $B^{2}=1 /\left.2\left(\partial \Gamma_{j}^{(1)-1} / \partial j\right)\right|_{j=0}$. Suppose the theorem true at order $j^{k}$ i.e., we have

$$
\begin{array}{r}
\frac{1}{2}\left(S^{j^{k}}, S^{j^{k}}\right)=O\left(j^{k+1}\right), \\
S^{j^{k}}=S+\sum_{n=1}^{k} j^{n} B^{n} .
\end{array}
$$

and

$$
B^{n}=\left.\frac{1}{n}\left(\partial^{n-1} \Gamma_{j^{n-1}}^{(1)-1} / \partial j^{n-1}\right)\right|_{j=0}
$$


At the regularized level, consider the action

$$
S_{\tau}^{j^{k}}=S_{\tau}+\sum_{n=1}^{k} j^{n} B_{\tau}^{n}
$$

and $\tilde{S}^{j^{k}}=S_{\tau}^{j^{k}}+\rho^{*} \theta_{\tau}^{j^{k}}$, with $\theta_{\tau}^{j^{k}}=\frac{1}{2 \tau}\left(S_{\tau}^{j^{k}}, S_{\tau}^{j^{k}}\right)+O\left(j^{k+1}\right)$, so that

$$
\frac{1}{2}\left(\tilde{S}^{j^{k}}, \tilde{S}^{j^{k}}\right)=\tau \frac{\partial \tilde{S}^{j^{k}}}{\partial \rho^{*}}+O\left(j^{k+1}\right)
$$

The corresponding equation for $\tilde{\Gamma}_{j^{k}}$ based on the action $\tilde{S}^{j^{k}}$ is

$$
\frac{1}{2}\left(\tilde{\Gamma}_{j^{k}}, \tilde{\Gamma}_{j^{k}}\right)=\tau \frac{\partial \tilde{\Gamma}_{j^{k}}}{\partial \rho^{*}}+O\left(j^{k+1}\right) .
$$

At one loop, we get, for the part independent of $\rho^{*}$,

$$
\left(S_{\tau}^{j^{k}}, \Gamma_{j^{k}}^{(1)}\right)=\tau \theta_{j^{k}}^{(1)}+O\left(j^{k+1}\right) .
$$

At order $j^{k}$, this equation gives

$$
\begin{aligned}
\left(S_{\tau},\left.\frac{\partial^{k} \Gamma_{j^{k}}^{(1)}}{\partial j^{k}}\right|_{j=0}\right)+\left(B_{\tau}^{1},\left.\frac{\partial^{k-1} \Gamma_{j^{k}}^{(1)}}{\partial j^{k-1}}\right|_{j=0}\right)+\ldots \\
+\left(B_{\tau}^{k},\left.\Gamma_{j^{k}}^{(1)}\right|_{j=0}\right)=\left.\tau \frac{\partial^{k} \theta_{j^{k}}^{(1)}}{\partial j^{k}}\right|_{j=0} .
\end{aligned}
$$

At order $1 / \tau$, we get, using

$$
\left.\frac{\partial^{n-1} \Gamma_{j^{k}}^{(1)-1}}{\partial j^{n-1}}\right|_{j=0}=\left.\frac{\partial^{n-1} \Gamma_{j^{n-1}}^{(1)-1}}{\partial j^{n-1}}\right|_{j=0}=n B^{n}
$$

for $n=1, \ldots, k-1$ and defining $\left.\frac{\partial^{k} \Gamma_{j^{k}}^{(1)-1}}{\partial j^{k}}\right|_{j=0}=(k+1) B^{k+1}$, the relation

$$
\left(S,(k+1) B^{k+1}\right)+\left(B^{1}, k B^{k}\right)+\ldots+\left(B^{k}, B^{1}\right)=0,
$$


or equivalently

$$
0=\sum_{m=0}^{k}\left(B^{m},(k+1-m) B^{k+1-m}\right)=\frac{(k+1)}{2} \sum_{m=0}^{k+1}\left(B^{m}, B^{k+1-m}\right),
$$

which proves the theorem.

Let $E^{0}=A_{1}=\theta^{(1)-1}-\left(B^{1}, S_{1}\right)$.

Theorem 4.4 The lowest order contribution to the anomaly $E^{0}$ can be extended to a local cocycle of the deformed solution of the master equation $S^{j^{\infty}}$, i.e., there exist local functionals $E^{m}$ such that

$$
\begin{array}{r}
\left(S^{j^{\infty}}, E^{j^{\infty}}\right)=0, \\
E^{j^{\infty}}=\sum_{m=0} j^{m} E^{m} .
\end{array}
$$

Proof. The theorem holds for $j^{0}$ and $j^{1}$ by taking in (3.9) $D=B^{1}$, and defining

$$
\begin{array}{r}
E^{1}=\left.\frac{\partial \theta_{j}^{(1)-1}}{\partial j}\right|_{j=0}-\left(D_{1}, \Gamma^{(1)-1}\right)-\left(D^{(1)-1}, S_{1}\right) \\
=\left.\frac{\partial \theta_{j}^{(1)-1}}{\partial j}\right|_{j=0}-\left(B_{1}, B_{1}^{1}\right)-\left(2 B^{2}, S_{1}\right) .
\end{array}
$$

Let us define

$$
E^{m}=\left.\frac{\partial^{m} \theta_{j^{m}}^{(1)-1}}{\partial j^{m}}\right|_{j=0}-\sum_{n=0}^{m}\left((n+1) B^{n+1}, B_{1}^{m-n}\right) .
$$

The consistency condition is

$$
\left(\Gamma_{j^{k}}, \frac{\partial \tilde{\Gamma}_{j^{k}}}{\partial \rho^{*}}\right)=O\left(j^{k+1}\right)
$$

At one loop, we have,

$$
\left(\Gamma_{j^{k}}^{(1)}, \theta_{\tau}^{j^{k}}\right)+\left(S_{\tau}^{j^{k}}, \theta_{j^{k}}^{(1)}\right)=O\left(j^{k+1}\right) .
$$


The term of order $j^{k}$ of this equation gives

$$
\sum_{m=0}^{k}\left[\left(\left.\frac{\partial^{m} \Gamma_{j^{k}}^{(1)}}{\partial j^{m}}\right|_{j=0},\left.\frac{\partial^{k-m} \theta_{\tau}^{j^{k}}}{\partial j^{k-m}}\right|_{j=0}\right)+\left(B_{\tau}^{m},\left.\frac{\partial^{k-m} \theta_{j^{k}}^{(1)}}{\partial j^{k-m}}\right|_{j=0}\right)\right]=0 .
$$

At order $1 / \tau$, we get

$$
\begin{gathered}
\sum_{m=0}^{k}\left[\left((m+1) B^{m+1}, \sum_{l=0}^{k-m}\left(B^{l}, B_{1}^{k-m-l}\right)\right)\right. \\
\left.+\left(B^{m},\left.\frac{\partial^{k-m} \theta_{j^{k}}^{(1)-1}}{\partial j^{k-m}}\right|_{j=0}\right)\right]=0 .
\end{gathered}
$$

Using the Jacobi identity, the first term is given by

$$
\sum_{m=0}^{k} \sum_{l=0}^{k-m}\left[\left(\left((m+1) B^{m+1}, B^{k-m-l}\right), B_{1}^{l}\right)-\left(B^{l},\left((m+1) B^{m+1}, B_{1}^{k-m-l}\right)\right)\right] .
$$

Changing the sum $\sum_{m=0}^{k} \sum_{l=0}^{k-m}$ to the equivalent sum $\sum_{l=0}^{k} \sum_{m=0}^{k-l}$, the first term of this equation vanishes on account of (4.3), while the second term, using the definition (4.6), combines with the second term of (4.7) to give

$$
\sum_{m=0}^{k}\left(B^{m}, E^{k-m}\right)=0
$$

which proves the theorem.

The investigation in this letters is a first step in order to analyze the cohomological restrictions on anomalies and counterterms at higher orders in $\hbar$. To see this, we note that if we put $j=(-\hbar / \tau)$, the action $S^{(-\hbar / \tau)^{\infty}}$ satisfies the (deformed) master equation $1 / 2\left(S^{(-\hbar / \tau)^{\infty}}, S^{(-\hbar / \tau)^{\infty}}\right)=0$, while the correponding effective action is finite at order $\hbar$. Its divergences at order $\hbar^{2}$ are poles up to order 2 in $\tau$ with residues that are local functionals. A systematic analysis of the substraction procedure at higher orders in $\hbar$ will be presented in [17].

\section{Acknowledgments}

The author wants to thank F. Brandt, M. Henneaux, J. Kottmann, M. Kreuzer, J. París and M. Tonin for useful discussions and Prof. H. Kleinert for hospitality in his group while this work has been completed. 


\section{References}

[1] C. Becchi, A. Rouet and R. Stora, Commun. Math. Phys. 42 (1975) 127 ; Ann. Phys. (NY) 98 (1976) 287.

[2] O. Piguet and S. P. Sorella, Algebraic Renormalization : Perturbative Renormalization, Symmetries and Anomalies, Lecture Notes in Physics vol 28, Springer Verlag 1995.

[3] J. Zinn-Justin, Renormalisation of gauge theories in Trends in elementary particle theory, Lecture notes in Physics $\mathrm{n}^{0} 37$ (Springer, Berlin 1975) ; J. Zinn-Justin, Quantum Field Theory and Critical Phenomena, $2^{\text {nd }}$ Edition, Clarendon Press (Oxford: 1993).

[4] I.A. Batalin and G.A. Vilkovisky, Phys. Lett. B102 (1981) 27 ; Phys. Rev. D28 (1983) 2567 ; Phys. Rev. D30 (1984) 508.

[5] P.S. Howe, U. Lindström and P. White, Phys. Lett. B246 (1990) 430.

[6] W. Troost, P. van Nieuwenhuizen and A. van Proeyen, Nucl. Phys. B333 (1990) 727.

[7] J. Wess and B. Zumino, Phys. Lett. B37 (1971) 95.

[8] D. Anselmi, Class. Quant. Grav. 11 (1994) 2181.

[9] J. Gomis and S. Weinberg, Nucl.Phys. B469 (1996) 473-487.

[10] H. Kluberg-Stern and J.B. Zuber, Phys. Rev. D12 (1975) 467, 482, 3159 .

[11] S. Weinberg, The Quantum Theory of Fields, Vol I and II, Cabridge University Press 1995, 1996.

[12] G. Barnich, F. Brandt and M. Henneaux, Commun.Math.Phys. 174 (1995) 93.

[13] M. Tonin, Nucl. Phys. (Proc. Suppl.) 29 (1992) 137. S. Ayoama and M. Tonin, Nucl. Phys. B179 (1981) 293.

[14] M. Henneaux and C. Teitelboim, Quantization of Gauge Systems, Princeton University Press (Princeton: 1992).

[15] J. Gomis, J. París and S. Samuel, Phys. Rep. 259 (1995) 1. 
[16] F. Brandt, M. Henneaux and A. Wilch, Extended antifield formalism and Ward identities for symmetries of higher order, preprint UB-ECMPF-97-7, hep-th/9705007.

[17] G. Barnich, in preparation.

[18] P.L. White Phys. Lett. B284 (1992) 55.

[19] F. De Jonghe, J. París and W. Troost, Nucl.Phys. B476 (1996) 559.

[20] J. París and W. Troost, Nucl.Phys. B482 (1996) 373.

[21] P. Breitenlohner and D. Maison, Commu. Math. Phys. 52 (1977) 11, 39,55 .

[22] G. Barnich and M. Henneaux, Phys. Lett. B 311 (1993) 123. 